\title{
Impact de la construction du port de Nouakchott (Mauritanie) sur l'évolution du trait de côte
}

\author{
Ahmed Ould ELMOUSTAPHA, Université de Nouakchott, FST \\ Franck LEVOY, Université de Caen, Unité CNRS 6143 M.2.C. \\ Hervé TEXIER, Université de Caen, Unité CNRS 6143 M.2.C.
}

\begin{abstract}
Résumé
Depuis la fin de la construction, en 1986, d'un port en eaux profondes sur la façade atlantique de Nouakchott, une évolution rapide du trait de côte est constatée. Une avancée, au Nord du port, de $35 \mathrm{~m}$ par an et une érosion annuelle de $25 \mathrm{~m}$ au sud ont été observées sur la période 1980-1998. Une étude des caractéristiques naturelles du milieu a permis de caractériser le fonctionnement hydrosédimentaire de ce littoral. Le code numérique UNIBEST (Delft Hydraulics, 1994) a été utilisé pour quantifier le transport sédimentaire et simuler l'évolution naturelle et anthropique du trait de côte dans la zone étudiée, permettant de faire ressortir l'impact morphodynamique des ouvrages portuaires.
\end{abstract}

\begin{abstract}
Along the coast of Mauritania, the building of a new harbour in 1986, close to Nouakchott, induced large shoreline position changes. Updriftward to the main jetty, accretion was observed with an annual rate of about $35 \mathrm{~m}$ per year. Downdriftward, intense shoreline recession $(35 \mathrm{~m} / \mathrm{y})$ contribute to dune destruction and flooding of low lying areas. Using the numerical model UNIBEST (Delft Hydraulics, 1994), simulations have been done to predict shoreline position with and without structures and also taking into account of a likely sea level rise acceleration.
\end{abstract}

\section{Introduction}

Située sur la façade Est de l'Océan Atlantique, Nouakchott, capitale de la Mauritanie, dispose d'un littoral relativement homogène d'un point de vue géomorphologique (fig. 1). Il est caractérisé par un transit sédimentaire longitudinal très important, orienté $\mathrm{N}-\mathrm{S}$, estimé d'après les études antérieures, entre 730000 et $950000 \mathrm{~m}^{3}$ par an (Mohameden, 1995, ITPT, 1998). La construction d'une digue portuaire d'environ $1300 \mathrm{~m}$ de long, entre 1979 et 1986 , a complètement perturbé le fonctionnement hydrosédimentaire du site et l'évolution du trait de côte. Ainsi, on constate :

- un fort engraissement de la côte au Nord du port ; 
- une érosion importante au sud des installations actuelles ayant déjà nécessité la mise en place de mesures de sauvegarde (épi, digue de retenue).

Les objectifs de cette étude sont d'améliorer la prévision de l'évolution du trait de côte de Nouakchott, et de la comparer à son évolution naturelle sans l'influence des aménagements, en tenant compte également d'une accélération éventuelle de l'élévation du niveau moyen de la mer.

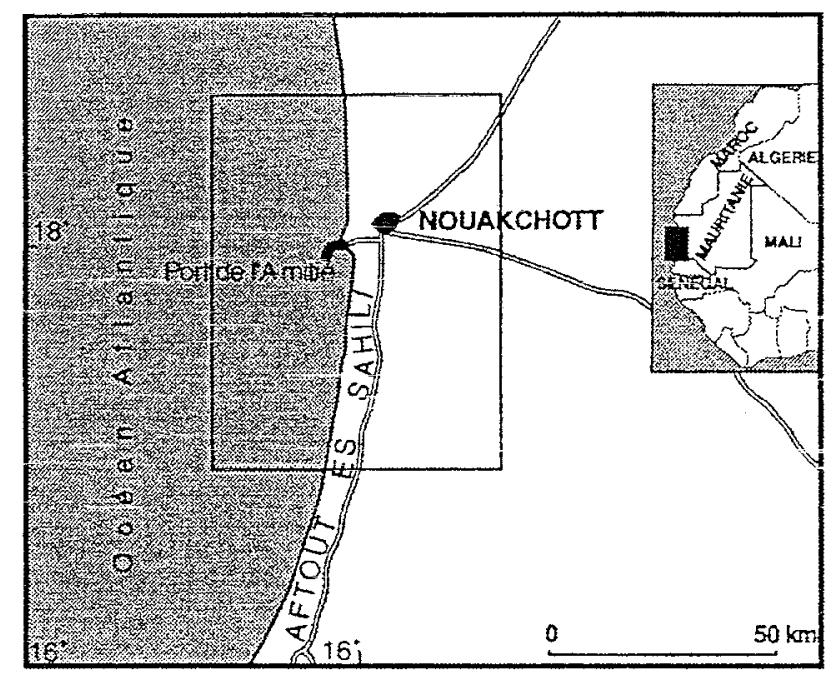

Figure 1 : Carte de localisation de la zone d'étude

Figure 1 : studied area

\section{Le cadre hydrodynamique général du littoral de Nouakchott}

\subsection{Les vents}

L'analyse des données météorologiques réalisée sur l'année 1996, au port de Nouakchott, montrent que les vents les plus fréquents proviennent des secteurs ENE à O. Les vents du secteur ENE à NNE représentent 40,6\% des conditions annuelles alors que les vents du secteur $\mathrm{N}$ à $\mathrm{O}$ sont présents dans $49,2 \%$ des situations. Les vents forts (supérieurs à $10.8 \mathrm{~m} / \mathrm{s}$ ) sont rares, représentant $2,3 \%$ des observations. Les vents inférieurs à $5,5 \mathrm{~m} / \mathrm{s}$ représentent $42,1 \%$ des conditions annuelles. $55,6 \%$ des vents ont des vitesses comprises entre 5,5 et $10,8 \mathrm{~m} / \mathrm{s}$.

\subsection{Les houles}

608 observations de houles ont été effectuées pendant une période d'une année, de mars 1996 à février 1997, par les Services Techniques du Port de l'Amitié.

L'étude de la houle sur 12 mois permet de constater que la hauteur significative moyenne est de 1.81 mètres. La majorité des houles est comprise entre 1 et 2.5 mètres, représentant plus de $90 \%$ des situations. Les périodes de pic se répartissent entre 6 et 23 secondes. Les agitations de périodes courtes, inférieures à $8 \mathrm{~s}$, sont rares, représentant moins de $5 \%$ des observations. Les directions de 
propagation dominantes sont comprises entre l'ENE et l'ONO. Elles représentent environ $90 \%$ des observations (fig. 2). Les hauteurs maximales sont associées aux houles des secteurs $\mathrm{N}$ à $\mathrm{O}$.

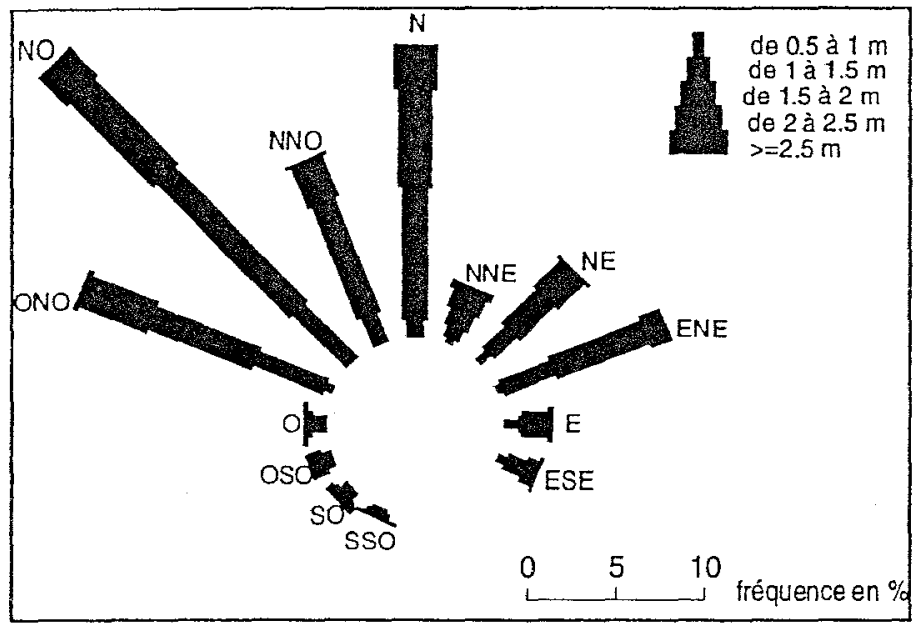

Figure $2:$ Rose de la houle à Nouakchott

Figure 2 : wave directional spectra close to Nouakchott harbour

\subsection{La marée}

La marée à Nouakchott est du type semi-diurne. Le marnage est de type microtidal avec :

- un niveau maximum de pleine mer de vive eaux de $2.05 \mathrm{~m}$;

- un niveau minimum observé de $0.03 \mathrm{~m}$ (Mohameden, 1995).

\subsection{Les courants généraux et induits par la houle}

Un seul grand courant océaniquie longe la côte mauritanienne : le courant des Canaries. Ce courant vient des régions tempérées. Une branche bifurque vers l'Ouest au niveau du Cap-Blanc, au Nord de la Mauritanie (Hebrard, 1973) pour former le courant Nord-Equiâturial qui baigne l'essentiel des côtes de la Mauritanie et du Sénégal. La vitesse de ce courant est en moyenne de 0,5 nœuds $(0,25 \mathrm{~m} / \mathrm{s})$.

Le courant d'obliquité à l'origine de la dérive littorale est généré principalement par la houle du Nord-Ouest. Il suit le rivage selon une direction générale NordSud. Des injections de la rhodamine ont été réalisées dans le déferlement, pour différents scénarios hydrodynamiques, dans le but de déterminer sa vitesse. Les mesures réalisées montrent qu'il peut dépasser $0.5 \mathrm{~m} / \mathrm{s}$.

\section{L'évolution historique du trait de côte}

L'évolution historique du littoral de Nouakchott entre 1980 et 1998 a été reconstituée en utilisant trois types de documents : la carte IGN (1980), les images 
satellitaires de 1987, 1989 et 1992 et les résultats de mesures concernant la position du trait de côte réalisées au DGPS en 1998. La figure 3 présente cette évolution.

Entre 1980 et 1998, une accumulation sableuse s'est développée immédiatement au Nord de l'ouvrage, contribuant à une avancée du trait de côte d'environ $670 \mathrm{~m}$ $(+35 \mathrm{~m} / \mathrm{an})$. Une érosion, atteignant $460 \mathrm{~m}$ en 18 ans, est constatée au Sud du port, correspondant à un recul moyen annuel d'environ $25 \mathrm{~m}$. Cette évolution peut être considérée comme exemplaire du fait de la simplicité du contexte hydrosédimentaire et des aménagements réalisés (effet d'épi).

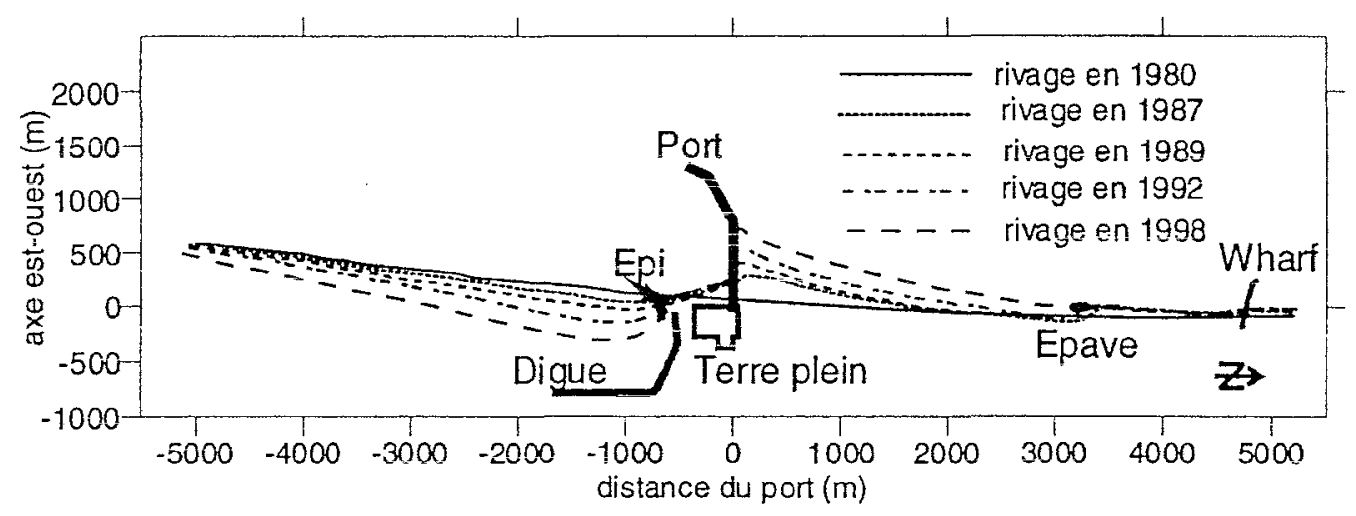

Figure 3 : évolution historique du trait de côte

Figure 3 : Historical shoreline location

\section{Présentation du modèle numérique utilisé}

Afin d'apprécier, d'une manière quantitative, l'impact de la construction du port de Nouakchott sur l'évolution du trait de côte, par rapport à l'évolution naturelle qui aurait pu être observée sans la réalisation du port, des simulations numériques ont été réalisées. Le logiciel UNIBEST (Delft Hydraulics, 1994) a été utilisé pour modéliser l'évolution du trait de côte sur une période pluri-décennale. Ce code de calcul repose sur la théorie de Pelnard-Considère $(1956,1986)$. Celle-ci s'applique à des zones côtières dont les évolutions transversales sont relativement lentes. Ce modèle s'adapte donc plutôt aux cas où l'évolution du trait de côte résulte de gradients longitudinaux de transports sédimentaires. Le cas du littoral de la Mauritanie est donc bien adapté à l'outil numérique compte tenu de l'existence d'une très forte dérive littorale.

Les variables prises en considération sont (GRESARC, 1996):

- les caractéristiques initiales de la géométrie de la côte (tracé en plan et bathymétrie) ;

- les caractéristiques granulométriques des sédiments;

- la hauteur, la direction et la période de la houle ;

- l'intensité et la direction des courants de marée ;

- la hauteur du plan d'eau statique. 
Les conditions hydrodynamiques sont, bien entendu, considérées comme invariantes sur le pas de temps considéré par les simulations.

\section{Choix des conditions de simulations}

\subsection{Condition initiale du modèle}

Le trait de côte initial choisi pour les calculs est celui de 1980, extrait de la carte IGN. A cette date, aucune structure portuaire ayant un impact morphosédimentaire n'est implantée.

Le tracé de la côte en 1980 montre une côte légèrement concave à très grand rayon de courbure.

\section{2 - Caractéristiques granulométriques}

L'observation de la plage de Nouakchott fait apparaître essentiellement du sable et accessoirement des galets. Des analyses granulométriques ont été réalisées à partir d'échantillons de sédiments prélevés le long d'un profil de la plage de Nouakchott. Il apparaît que la granulométrie varie sur le site entre 0,18 à $0,37 \mathrm{~mm}$ transversalement au trait de côte. Une granulométrie moyenne caractérisée par un $D_{50}$ et un $D_{90}$, respectivement égales à $0.21 \mathrm{~mm}$ et $0.38 \mathrm{~mm}$, a été prise en compte pour caractériser les sédiments de la partie supérieure de l'estran. La vitesse de chute du sédiment retenue pour le calcul numérique est de $2.1 \mathrm{~cm} / \mathrm{s}$.

\section{3 - Caractéristiques bathymétriques}

Le profil transversal de la partie active du profil de la plage, utilisé pour effectuer les simulations numériques, a été défini à partir de mesures réalisées en septembre 1998. La pente moyenne du profil est estimée à $5 \%$. Pour assurer la propagation des houles du point de mesures, vers le haut estran, le profil est complété dans sa partie inférieure par des données extraites de la carte bathymétrique et topographique réalisée par la mission chinoise de prospection du port en 1975.

\subsection{Conditions d'agitation}

Les premiers tests de sensibilité du modèle réalisés indiquent que la marée, avec un marnage d'environ $2 \mathrm{~m}$ au maximum, peut être ignorée lors des phases de simulation, pour les pas de temps longs, supérieurs à une année. Un plan d'eau moyen peut donc être utilisé. Les courants de marée, généralement très faibles, ont une incidence négligeable sur les débits solides. Ils peuvent également être ignorés. La définition du climat d'agitation est essentielle. Sept principales directions de provenance ont été identifiées entre les secteurs NNO et SSO. Les houles de quadrant est, mesurées au large, sont ignorées dans la mesure où, à la côte, celles-ci arrivent réfractées, provenant du NNO ou du NO. En ce qui 
concerne le choix des hauteurs et des périodes associées, des calculs préliminaires ont été réalisés. Ils ont permis de mettre en évidence que l'écart de débits solides calculés d'une part, à partir de la moyenne annuelle des hauteurs et des périodes pour une direction de provenance de la houle et d'autre part, à partir d'une somme des débits solides pour des scénarios associant des classes de hauteurs et de périodes distinctes observées sur une année, était faible. La première méthode surestime les débits solides de l'ordre de $15 \%$. Afin de limiter le nombre de simulations, une moyenne des hauteurs de houle et des périodes pour une direction de provenance a été retenue. Le tableau 1 présente les caractéristiques de l'agitation prise en compte pour les simulations réalisées.

\begin{tabular}{ccccc}
\hline $\begin{array}{c}\text { Niveau moyen } \\
\text { de l'eau }\end{array}$ & $\begin{array}{c}\text { Direction de la } \\
\text { houle }\end{array}$ & $\begin{array}{c}\text { Période de } \\
\text { Pic(s) }\end{array}$ & $\begin{array}{c}\text { Hauteur } \\
\text { significative }(\mathrm{m})\end{array}$ & Durée (jours) \\
\hline 0.97 & NNO & 8.3 & 1.79 & 33 \\
0.97 & NO & 7.96 & 1.64 & 168.7 \\
0.97 & ONO & 9.35 & 1.92 & 86 \\
0.97 & O & 11.28 & 2.26 & 44.8 \\
0.97 & OSO & 7.9 & 1.7 & 10.6 \\
0.97 & SO & 6.5 & 1.23 & 8.1 \\
0.97 & SSO & 6.03 & 1.28 & 2.4 \\
\hline
\end{tabular}

Tableau 1 : Climat d'agitation annuel utilisé pour les simulations numériques Table I : wave conditions used for modelling

\section{Le calage du modèle et conditions aux limites}

Pour ajuster le calage, il est possible d'agir sur les conditions aux limites longitudinales du modèle et de régler ainsi les transports sédimentaires entrants dans le système. La condition aux limites choisie suppose que la côte conserve une orientation constante au cours du temps sur les points frontières considérés. Cette condition implique, en particulier, que le transport sédimentaire soit également invariable au cours du temps en ce point. Cette condition s'adapte bien au présent contexte. En effet, aux limites nord et sud de la zone étudiée, une faible variation de l'orientation du trait de côte avec le temps a été observée au cours de l'évolution historique du site (fig.3).

Le calage du modèle repose essentiellement sur la connaissance de l'évolution historique du trait de côte. Une comparaison est donc effectuée entre le rivage calculé et le rivage constaté pour les années 1987 et 1998. La figure 4 présente le trait de côte de Nouakchott mesuré et calculé en 1998 en tenant compte de la présence des infrastructures portuaires.

Les écarts entre les rivages calculés et ceux mesurés sont relativement faibles. La tendance évolutive de la côte est globalement bien décrite sur 18 ans (1980 1998) et la position des traits de côte intermédiaires est correctement simulée. 


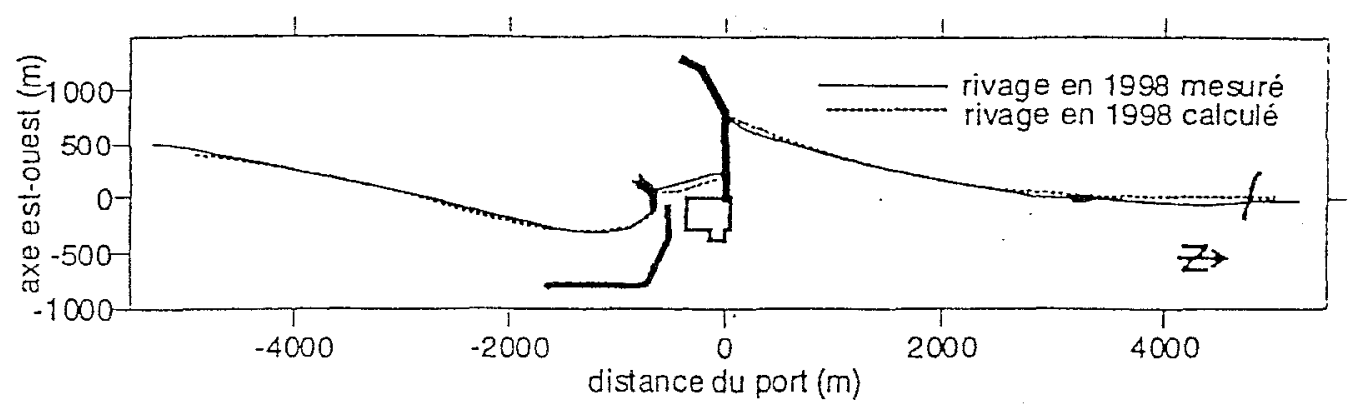

Figure 4 : Comparaison entre la position du trait de côte calculé et mesuré en 1998 Figure 4: Comparison between calculated and measured shoreline location

\section{Tests préliminaires}

\subsection{Influence du choix de la formule de transport}

A partir des données morphosédimentaires et hydrodynamiques, une estimation des débits solides a pu être effectuée pour choisir la formule de transport la plus appropriée au site étudié. Les calculs réalisés avec UNIBEST montrent que les écarts entre les débits solides calculés peuvent être considérables, variant de 1 à 6 . Les formules utilisées par le L.N.H. (Bonnefille, 1980) et le L.C.H.F. (Migniot, 1977 et 1990) indiquent également des résultats variant d'un facteur 1 à 2, compris entre 871000 et $1814000 \mathrm{~m}^{3}$. Par référence aux informations issues de la littérature concernant le transit sédimentaire longshore, la formule de Baillard ${ }^{1}$ (1981) a été utilisée. Cette formule donnent les débits solides les plus faibles, mais surtout les plus réalistes, par rapport aux estimations des débits issues de la littérature (Mohameden, 1995).

\subsection{Influence du choix de la hauteur dü profil actif}

La profondeur $\mathrm{P}$ de fermeture du profil de plage (limite d'action des houles) est un paramètre important conditionnant fortement l'évolution du trait de côte (GRESARC, 1996). Plus la valeur de P est grande, plus le déplacement du trait de côte est faible.

Dans le présent travail, la comparaison entre les rivages calculés et les rivages mesurés au cours de la période 1980-1998 a montré qu'une valeur de P de 10 mètres permet d'obtenir des résultats satisfaisants.

\section{Evolution prévisionnelle du trait de côte}

\subsection{L'évolution naturelle}

L'évolution naturelle, sans aménagement, du trait de côte de Nouakchott a ainsi d'abord été simulée à partir de l'état initial de 1980 sans tenir compte d'une

\footnotetext{
${ }^{1}$ Formule décrite en fin d'article
} 
élévation du niveau moyen de la mer. La figure 5 révèle que la côte de Nouakchott serait une zone d'accumulation. Ce gain de terre serait variable sur la portion de côte étudiée, allant de $65 \mathrm{~m}$ au Sud, à environ $5000 \mathrm{~m}$ de l'implantation actuelle du port, à $175 \mathrm{~m}$ à environ $3500 \mathrm{~m}$ au Nord. La progression du trait de côte aurait ainsi variée de 1 à $3 \mathrm{~m} / a n$ selon les endroits. Les transports sédimentaires sont pratiquement invariants au cours du temps, compris entre $825000 \mathrm{~m}^{3}$ au Sud à $1100000 \mathrm{~m}^{3}$ au Nord. L'influence de l'incidence de l'approche des houles à la côte est mise en évidence, plus forte au Nord, elle induit un transport plus important, que sur la partie sud. L'évolution du trait de côte résultant de gradients longitudinaux de transport sédimentaire, dans ces conditions, il est logique de constater un engraissement de la côte de Nouakchott.

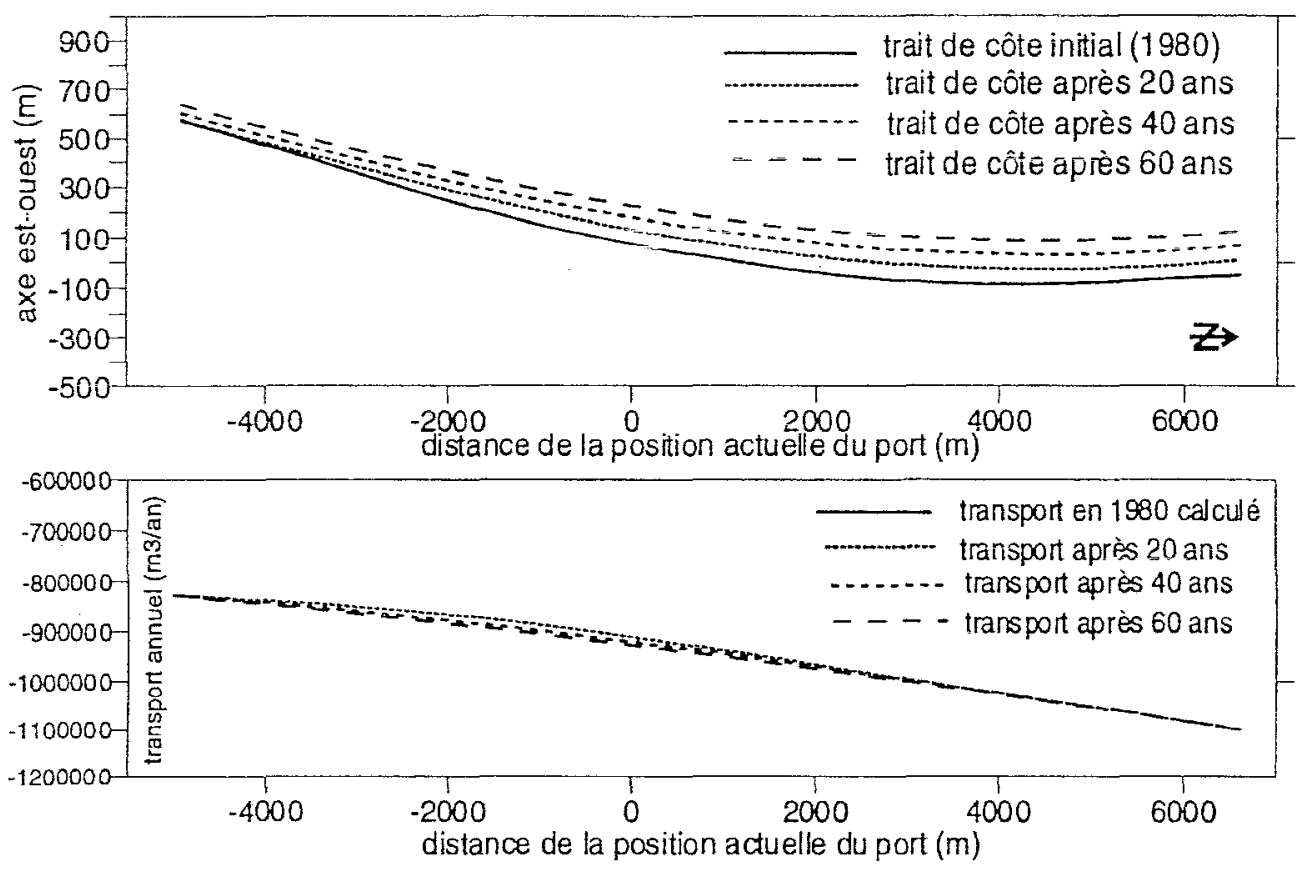

Figure 5 : Simulation de l'évolution du littoral de Nouakchott à partir de 1980 sans aménagement

Figure 5 : Simulations of shoreline evolution from 1980 without structure influences

\subsection{Influence de l'élévation du niveau marin sur l'évolution du trait de côte}

L'évolution naturelle du site a été simulée sur une période de 50 ans, en considérant une élévation du niveau moyen de la mer de $0.3 \mathrm{~m}$ (SCOR Working Group 89, 1991), avec un pas de temps de 10 ans. Il s'avère que :

- au Nord de la zone d'étude, l'influence de l'élévation du niveau moyen de la mer n'est pas sensible ;

- au Sud, l'avancée du trait de côte observée sans élévation du niveau moyen de la mer est réduite au maximum de $8 \mathrm{~m}$ sur 50 ans, soit une diminution de $12 \%$ 
de la progression du trait de côte traduisant une légère augmentation des débits solides le long de la frontière sud de la zone d'étude.

Il apparaît ainsi, que l'élévation du niveau moyen de la mer aurait un impact pratiquement insignifiant à l'échéance de 50 ans sur ce type de côte dominé par une dérive sédimentaire longitudinale.

\subsection{Impact des ouvrages portuaires existants sur l'évolution du trait de côte}

Une prospective d'évolution a été calculée en présence des ouvrages existants (fig.6). L'évolution induite par la présence du port est considérable, confirmant l'évolution historique observée sur la période 1980-1998 (fig.7). Au Nord des infrastructures, le transport sédimentaire varie d'environ $1000000 \mathrm{de}^{3}$ par an, à une valeur nulle au niveau de la digue-épi, la totalité du transit sédimentaire longshore étant stoppée, au moins jusqu'en 2010. La progression du trait de côte est d'environ $35 \mathrm{~m} /$ an jusqu'en 2005 et de $16 \mathrm{~m} /$ an entre 2015 et 2025 , le contournement de l'obstacle s'effectuant au cours de cette période.

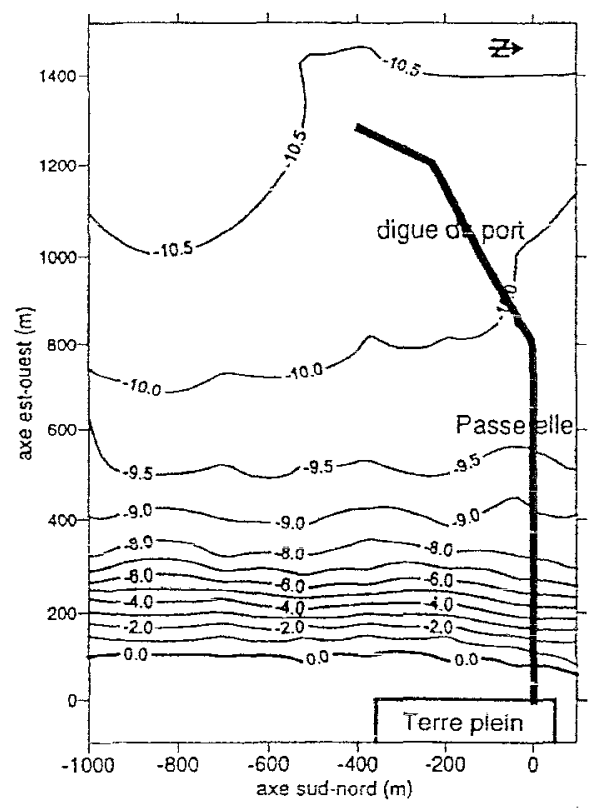

Figure 6: Carte bathymétrique de l'avant côte aux environs du poit d'après les relevés de la Mission Chinoise en 1975

Figure 6: Bathymetric map of the shoreface close to the Nouakchott Port

Au Sud du port, le déficit sédimentaire est considérable. Le gradient croissant des débits solides du Nord vers le Sud induit une érosion qui atteint près de $26 \mathrm{~m} / \mathrm{an}$ sur la période 2005 à 2025. 

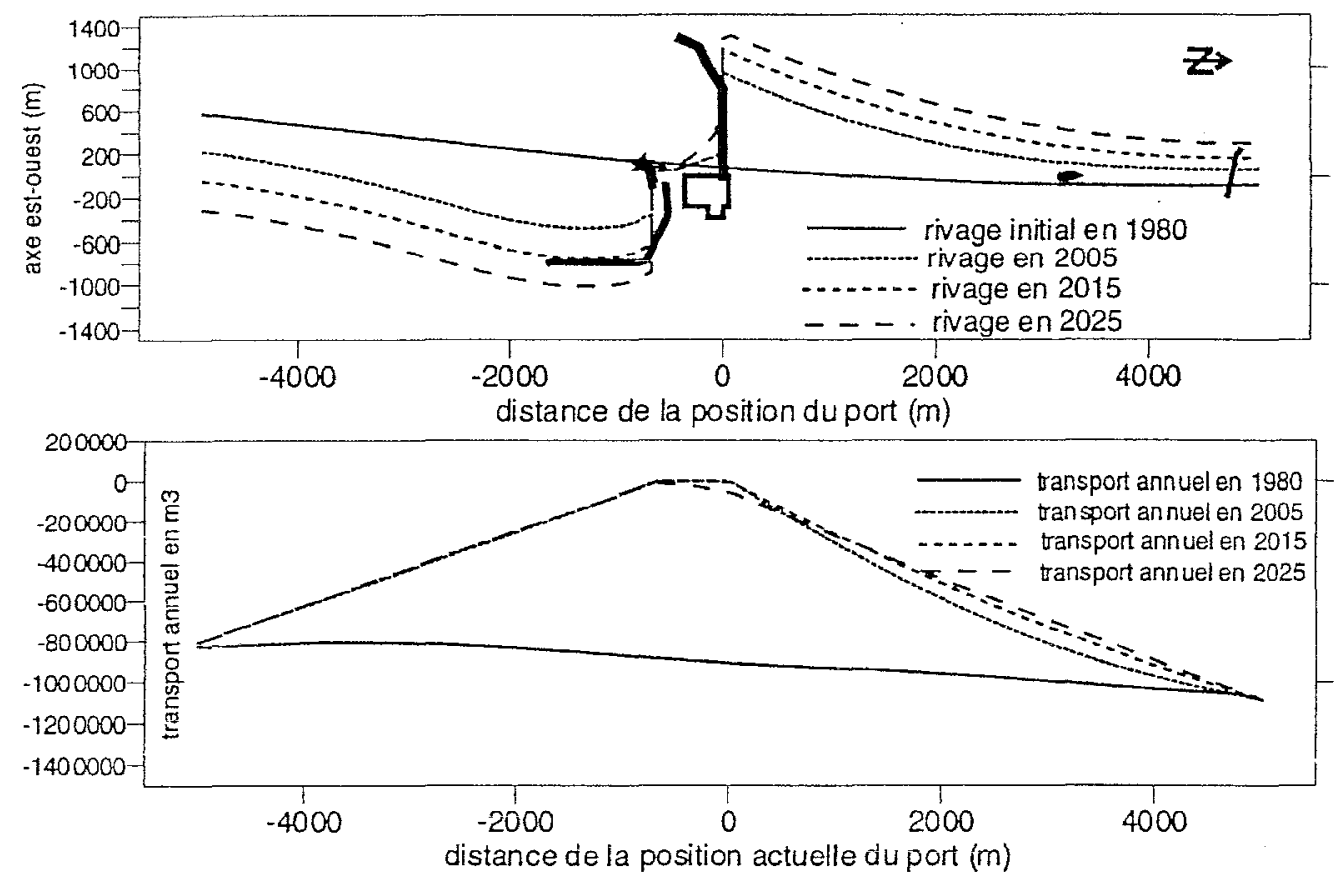

Figure 7 : Simulation de l'évolution du littoral de Nouakchott à partir de 1980 en tenant compte des aménagements

Figure 7 : Simulation of shoreline locations from 1980 to 2025 with structure influences

\section{Conclusions}

La campagne de mesures menée à proximité du port de Nouakchott, en 1996, a permis d'élaborer une base de données concernant les caractéristiques des houles sur une période de 12 mois. Complétée par des informations morphologiques et sédimentologiques, ces données ont été utilisées pour réaliser des simulations numériques de l'évolution du trait de côte qui aurait été observée sans la réalisation des aménagements portuaires de Nouakchott. Ainsi, une côte qui aurait du être en accrétion à un rythme d'environ 1 à $3 \mathrm{~m} /$ an, se trouve aujourd'hui en partie en érosion avec des taux de recul de plusieurs dizaines de mètres par an et des taux d'accrétion du même ordre de grandeur en valeur absolue. L'anthropisation du littoral de Nouakchott a donc amplifié le rythme de son évolution d'un facteur 10 .

\section{Références}

Bonnefille R., 1980. Cours d'Hydraulique Maritime. ENSTA. Ed. Masson. 159 p.

DELFT HYDRAULICS, 1994. A Software Suite for Simulation of Sediment Transport Processes and related Morphodynamics of Beach Profiles and Coastline Evolution, Theorical reference document. $124 \mathrm{p}$. 
GRESARC, 1996. Conséquences d'une élévation du niveau moyen de la mer sur l'évolution d'un trait de côte sableux, Ministère de l'environnement. 79 p.

Hebrard L., 1973. Contribution à l'étude géologique du quaternaire du littoral mauritanien entre Nouakchott et Nouadhibou. Participation à l'étude des désertifications du Sahara. Thèse de l'Université de Lyon. 483 p.

ITPT, 1998. Projet de construction d'un port de pêche à Nouakchott, rapport final global. Ministère des Pêches et de l'Economie Maritime, Mauritanie. $78 \mathrm{p}$.

Mohameden A. O., 1995. Aménagement et évolution du littoral, cas du port de Nouakchott. Thèse de doctorat de l'Université de Nice. 149 p.

Migniot C., 1977. Action des courants et de la houle sur les sédiments. La Houille Blanche, $n^{\circ} 1$. pp 9-47.

Migniot C., 1990. Manuel d'hydrodynamique sédimentaire. S.T.C.P.M.V.N.159 p.

Pelnard-Considère R., 1956. Essai de la théorie de l'évolution des formes de rivage en plages de sable et galets. Soc. Hydro-technique de France, IV Journées de l'Hydraulique, Les énergies de la mer, Paris, France.

Pelnard-Considère R., 1986. Essai de théorie de l'évolution des formes de rivage en plages de sables et de galets. XVIII journées de l'hydraulique, S.H.F. Marseille.

SCOR Working Group 89, 1991. The Response of Beaches to Sea-Level Changes: A Review of Predictive Models. Iournal of Coastal Research, Vol. 7, $N^{0} 3$, pp 895-921.

La loi de transport sédimentaire utilisée, sous l'interaction des houles et des courants, dérinie par Baillard en deux dimensions dans un plan horizontal (Baillard, 1981) s'écrit sous la forme:

$$
\begin{aligned}
& q_{x}=\frac{c_{f}}{\Delta g N} \frac{\varepsilon_{B}}{\tan \phi}\left[\left\langle\left. u\right|^{2} u_{x}\right\rangle-\frac{\tan \beta_{x}}{\tan \phi}\left\langle|u|^{3}\right\rangle\right]+\frac{c_{f}}{\Delta g N} \frac{\varepsilon_{s}}{w}\left[\left\langle\left. u\right|^{3} u_{x}\right\rangle-\frac{\varepsilon_{s}}{w} \tan \beta_{x}\left\langle|u|^{5}\right\rangle\right] \\
& q_{y}=\frac{c_{f}}{\Delta g N} \frac{\varepsilon_{B}}{\tan \phi}\left[\left\langle\left. u\right|^{2} u_{y}\right\rangle-\frac{\tan \beta_{y}}{\tan \phi}\left\langle|u|^{3}\right\rangle\right]+\frac{c_{f}}{\Delta g N} \frac{\varepsilon_{s}}{w}\left[\left\langle\left. u\right|^{3} u_{y}\right\rangle-\frac{\varepsilon_{s}}{w} \tan \beta_{y}\left\langle|u|^{5}\right\rangle\right]
\end{aligned}
$$

où:

$\mathrm{q}_{x}$ et $\mathrm{q}_{y}$ : sont les composantes suivant les directions $x$ et $y$ du vecteur de transport global; $\mathrm{u}_{\mathrm{x}}$ et $\mathrm{u}_{\mathrm{y}}$ : sont les composantes suivant les directions $\mathrm{x}$ et $\mathrm{y}$ de la vitesse instantanée près du fond

$\tan \beta_{x}=\frac{\partial z_{b}}{\partial x} \quad \tan \beta_{y}=\frac{\partial z_{b}}{\partial y} \quad z_{b}=$ profondeur du fond

$\Delta:$ la densité relative du sédiment

$\mathrm{N}$ : la porosité des fonds tan $\phi:$ l'angle de frottement interne

w : la vitesse de chute des sédiments $\varepsilon_{\mathrm{f}}$ : le coefficient de frottement $\varepsilon_{\mathrm{b}}$ : le facteur de rendement du transport par charriage $\varepsilon_{\mathrm{s}}:$ le facteur de rendement du transport en suspension 